\title{
Review of Published Case Reports of COVID-19 Associated Mucormycosis with Search of Therapeutic Potential in Ayurveda and Homeopathy
}

\author{
Danish Javed ${ }^{1}$, Ashish Kumar Dixit ${ }^{1}$, Himanshi Vats ${ }^{2}$, Sana Anwar ${ }^{3}$, Nibha Giri ${ }^{4}$ \\ ${ }^{1}$ Department of AYUSH, All India Institute of Medical Sciences, Bhopal, Madhya Pradesh, India \\ ${ }^{2}$ Department of Shalya, BMK KLE University, Belgavi, Karnataka, India \\ ${ }^{3}$ Department of Oral Pathology \& Microbiology, Institute of Dental Sciences and Technology, Modinagar, \\ Uttar Pradesh, India \\ ${ }^{4}$ State Homeopathic Dispensary, Jakhanian, Gazipur, Uttar Pradesh, India
}

Corresponding Author: Danish Javed

\begin{abstract}
Background and Objectives: Infection with COVID-19 has been linked to fungal diseases. The sudden increase in mucormycosis cases has sparked the scientific community interest. In this review, we attempted to investigate the role of Ayurveda and Homoeopathy in the treatment of mucormycosis.

Methodology: The research was carried out in three stages; first step, studies on COVID-19 and mucormycosis were searched on various databases such as PubMed and Google Scholar until May 21, 2021, second step was to search authentic Ayurvedic and Homeopathic text books for the context of mucormycosis and symptoms were repertorised to find homeopathic medicines and finally, in the last step, findings were analysed and concluded in order to arrive at a factual solution.

Result: We found total 16 search results from bio-medical databases of which 14 articles were included. Except source books, no studies with respect to Ayurveda and Homoeopathy were found in above databases. In Ayurveda, Rajyakshama and krimijshirorog has close similarity to COVID associated mucormycosis. Vrinhana, vata-pitta nashakmadhuraushadhi, gritpaan,dugdha, nasya, dhum, kawal, gandush, mriduvirechan may be beneficial along with immune boosting drugs like ashawagandha, giloya, tulsi, vidanga etc. Rhino-orbito-cerbral mucormycosis (ROCM) can be treated with Arsenic album, Kali bichromicum, and Merc solubilis, while pulmonary mucormycosis (PM) with Arsenic album, Phosphorus and Acalypha indica. Similarly, gastrointestinal mucormycosis (GM) and cutaneous mucormycosis (CM) can be combated with Arsenic album, Lachesis, Merc Corrosivus etc. and Anthracinum, Arsenic album, Lachesis, Sulphur, Secale cornutum, and so on in Homoeopathy.

Conclusion: In the absence of rigorous trials, the review results found no conclusive comment on the efficacy of CAM in mucormycosis, but anecdotal evidence suggests in favour. To demonstrate its role, rigorous, exploratory, pilot, randomised control trials should be conducted in the future, either alone or as an add-on.
\end{abstract}

Key Words: COVID-19; Mucormycosis; Black Fungus; Post-COVID-19 care; Rehabilitation; Ayurveda; Homoeopathy

\section{INTRODUCTION}

Coronavirus disease has become a global challenge as the SARS COV-2 virus is changing itself day by day through multiple mutations and new variants are becoming troublesome [1]. COVID-19 infection has been seen to be associated with fungal diseases. Sudden upsurge of mucormycosis cases has focused the attention of the scientific community. 
Mucormycosis is caused by a group of fungi belonging to the order Mucorales. It is an angioinvasive disease mainly involves Rhizopus, Mucor, Rhizomucor, Cunninghamella and Absidia. The frequent disease causing organism is Rhizopus species, which has been reported $46 \%$ mortality in patients [2]. Mucormycosis is not a new disease; however the sudden rise in these cases has been seen during COVID-19 time. Its prevalence in India has been noted 0.14 cases per 1000 , which is 80 times more as compared to developed countries [3]. Total 8,848 cases of COVID-19 associated mucormycosis have been reported in India till May 22, 2021.

Many theories are being correlating the link of this incidence with COVID 19. Its prevalence in immune-compromised post-COVID-19 patients, who were on long term steroids, remdesivir, tocilizumab, oxygen support especially on industrial or contaminated sources of oxygen, contact with dirty hospital linen etc, has wide room for research. The complicated cases of orbital and cerebral involvement are more related to uncontrolled diabetes and diabetic ketoacidosis along with the unjustified use of high dose steroids. In India, diabetes mellitus is the most common risk factor related to mucormycosis [4]. As India has higher prevalence of mucormycosis and diabetes, it has become world capital of COVID-19 associated mucormycosis. Hyperglycemia has been found to augment the endothelial receptor GRP78, consequently leading to PMN cells dysfunction, impaired chemotaxis and defective intracellular death. High blood levels of zinc and iron have also shown intracellular affinity of fungal agents.

Immuno-compromised state of COVID-19 patients is susceptible to many opportunistic fungal infections. Till date in India, cases of Black fungus, white fungus and yellow fungus have been reported. Antifungal treatment is available in very limited amounts. Liposomal Amphotericin $\mathrm{B}$, Isavuconazole and Posaconazole are being currently used as antifungal agents for its treatment along with the extensive surgical debridement.

Ayurveda is catching its attention in this scenario and may provide some better outcome if used along with standard treatment. Recently, some authorities jointly started an initiative to manage the cases of mucormycosis with the help of Ayurveda medicines [5]. The high risk of mucormycosis or black fungus on recovering COVID-19 patients can be taken the edge off with prophylactic AYUSH medicines to strengthen their immunity. It was also proposed to prepare an adjuvant Ayurveda medicines kit for such patients and will be recommended in hospitals along with the standard care after getting proper approvals [6].

Here, we have performed wide search and analysis of various literature including journal articles, texts available in ayurveda, and homeopathy related and analogous to mucormycosis. The possibilities of integration of AYUSH with mainstream has been tried to explore in this pandemic situation that may open the new path of success and knowledge.

\section{METHODOLOGY}

The study was performed in three steps. In the first step, studies related to COVID-19 and mucormycosis was searched on various database including PubMed, Google Scholar till $21^{\text {st }}$ may, 2021 using key words "COVID-19", "mucormycosis", and "Black fungus" with Boolean operators "AND"/ "OR". Their findings were analyzed and symptoms, diagnostic methods, presentation, available treatment methods of COVID-19 associated mucormycosis were assessed in systematic manner. In the second steps, authentic text books of Ayurveda and Homeopathy were also searched to find the context of similar features of post COVID-19 mucormycosis. Repertorisation of symptoms was performed to find out the possible solution through Homeopathic medicines with the help of software Homoeopathic Repertorium version 3.9.5.7. In the third step, all the 
findings were analyzed and concluded in a manner to put some factual solution.

\section{RESULT}

\subsection{Studies related to COVID-19 and mucormycosis}

Table 1: Details of Case Reports/Series of mucormycosis

\begin{tabular}{|c|c|c|c|c|c|c|c|c|c|c|}
\hline SN & Study & Subject & $\begin{array}{l}\text { Case } \\
\text { presentation }\end{array}$ & $\begin{array}{l}\text { Associated } \\
\text { co-morbidity }\end{array}$ & Diagnosis & Investigation & Fungus & $\begin{array}{l}\text { Anti-fungal } \\
\text { Treatment }\end{array}$ & $\begin{array}{l}\text { Surgical } \\
\text { management }\end{array}$ & Outcome \\
\hline 1 & $\begin{array}{l}\text { Maini } \\
\text { A et } \\
\text { al.2021 } \\
\text { (7) }\end{array}$ & 1 & $\begin{array}{l}\text { 38-year-old } \\
\text { male with } \\
\text { swelling and } \\
\text { pain in the left } \\
\text { eye }\end{array}$ & $\begin{array}{l}\text { Inj.Remdesivi } \\
\mathrm{r} \\
\text { +InjMethylpre } \\
\text { dnisolone } \\
\text { +Inj.Dexamet } \\
\text { hasone }\end{array}$ & $\begin{array}{l}\text { Sino-orbital } \\
\text { mucormyco } \\
\text { sis }\end{array}$ & MRI & $\begin{array}{l}\text { Rhizopus } \\
\text { oryzae }\end{array}$ & $\begin{array}{l}\text { Inj.Flucona } \\
\text { zole 200 } \\
\text { mg- } \\
\text { 12hourly \& } \\
\text { Inj. } \\
\text { Amphoteric } \\
\text { in B 300 } \\
\mathrm{mg} / \text { day, }\end{array}$ & $\begin{array}{l}\text { Functional } \\
\text { Endoscopic } \\
\text { Sinus Surgery } \\
\text { (FESS), } \\
\text { Surgical } \\
\text { debridement }\end{array}$ & Improved \\
\hline 2 & $\begin{array}{l}\text { Payam } \\
\text { Tabarsi } \\
, 2021 \\
(8)\end{array}$ & 1 & $\begin{array}{l}50 \text {-year-old } \\
\text { woman with } \\
\text { facial swelling, } \\
\text { facial numbness, } \\
\text { periorbital } \\
\text { edema, } \\
\text { headache and } \\
\text { erythema }\end{array}$ & $\begin{array}{l}\text { Type } 2 \text { DM } \\
\text { and } \\
\text { hypertension, } \\
\text { five years ago } \\
\text { +gastric } \\
\text { bypass } \\
\text { surgery } \\
\text { +remdesivir } \\
\text { +dexamethaso } \\
\text { ne }\end{array}$ & $\begin{array}{l}\text { Rhinosinusi } \\
\text { tis }\end{array}$ & $\begin{array}{l}\text { Nasal } \\
\text { endoscopy, } \\
\text { PNS CT, } \\
\text { tissue } \\
\text { biopsies, PCR } \\
\text { and DNA } \\
\text { sequencing }\end{array}$ & $\begin{array}{l}\text { Rhizopus } \\
\text { oryzae }\end{array}$ & $\begin{array}{l}\text { Inj. } \\
\text { liposomal } \\
\text { amphoterici } \\
\text { n B }\end{array}$ & $\begin{array}{l}\text { Surgical } \\
\text { evaluation }\end{array}$ & Improved \\
\hline 3 & $\begin{array}{l}\text { Khan } \\
\text { N, et } \\
\text { al, } \\
2020 \\
(9)\end{array}$ & 1 & \begin{tabular}{lr}
\multicolumn{2}{l}{ 44-year-old } \\
woman with \\
COVID & 19 \\
pneumonia &
\end{tabular} & $\begin{array}{l}\text { Type } 2 \text { DM } \\
\text { +remdesivir } \\
\text { +methylpredn } \\
\text { isolone }\end{array}$ & $\begin{array}{l}\text { Pulmonary } \\
\text { mucormyco } \\
\text { sis }\end{array}$ & $\begin{array}{l}\text { CT Chest, } \\
\text { Bronchoscopy } \\
\text { Endobronchia } \\
1 \text { biopsy and } \\
\text { bronchoalveol } \\
\text { ar lavage } \\
\text { (BAL) }\end{array}$ & $\begin{array}{l}\text { Candida } \\
\text { albicans, } \\
\text { glabrata, } \\
\text { and } \\
\text { krusei } \\
\text { along } \\
\text { with } \\
\text { Aspergill } \\
\text { us flavus } \\
\text { and niger }\end{array}$ & $\begin{array}{l}\text { Voriconazol } \\
\mathrm{e} 250 \mathrm{mg} \\
\text { IV, } \\
\text { Micafungin } \\
150 \mathrm{mg} \mathrm{IV}\end{array}$ & & Death \\
\hline 4 & $\begin{array}{l}\text { Mishra } \\
\text { N, } \\
2021 \\
(10)\end{array}$ & 10 & $\begin{array}{l}\text { Mean age } 55.8 \\
\text { years ( } 37 \text { to } \\
78), 9 \text { male } 1 \\
\text { female with Eye } \\
\text { pain, facial pain } \\
\text { and nasal block }\end{array}$ & $\begin{array}{l}\text { DM (8), } \\
\text { and/or HTN, } \\
\text { CKD } \\
\text { +steroids(6) } \\
\text { +tocilizumab } \\
\text { (1) } \\
\text { +Remdesivir( } \\
\text { 5) }\end{array}$ & $\begin{array}{l}\text { Rhino- } \\
\text { orbital- } \\
\text { cerebral } \\
\text { mucormyco } \\
\text { sis }\end{array}$ & $\begin{array}{ll}\text { CT PNS, MRI } \\
\text { brain, HPE } \\
\text { and fungal } \\
\text { smear }\end{array}$ & $\begin{array}{l}\text { Rhizopus } \\
\text { species }\end{array}$ & $\begin{array}{l}\text { IV } \\
\text { Amphoteric } \\
\text { in B }\end{array}$ & $\begin{array}{l}\text { FESS, } \\
\text { debridement, } \\
\text { Orbitalexenter } \\
\text { ation,Endosco } \\
\text { picmaxillecto } \\
\text { my and } \\
\text { ethmiodectom } \\
\text { y }\end{array}$ & Death(4) \\
\hline 5 & $\begin{array}{l}\text { Kirill } \\
\text { A, et al } \\
2021 \\
(11)\end{array}$ & 1 & $\begin{array}{l}\text { 41-year-old man } \\
\text { hospitalized for } \\
\text { COVID } 19\end{array}$ & $\begin{array}{l}\text { T1DM } \\
\text { +diabetic } \\
\text { ketoacidosis } \\
\text { +steroids } \\
\text { +hydroxychlo } \\
\text { roquine }\end{array}$ & $\begin{array}{l}\text { Rhino- } \\
\text { cerebral- } \\
\text { mucormyco } \\
\text { sis }\end{array}$ & $\begin{array}{l}\text { CT,MRI } \\
\text { Brain }\end{array}$ & $\begin{array}{l}\text { Rhizopus } \\
\text { species }\end{array}$ & $\begin{array}{l}\text { Amphoteric } \\
\text { in } \\
\text { (abelcet) }\end{array}$ & 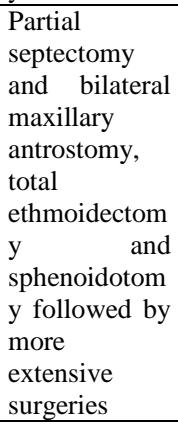 & Improved \\
\hline 6 & $\begin{array}{l}\text { Garg d, } \\
\text { et al, } \\
2021 \\
(12)\end{array}$ & 1 & $\begin{array}{l}55 \text {-year-old man } \\
\text { with severe } \\
\text { COVID } 19\end{array}$ & $\begin{array}{l}\text { diabetes } 10 \\
\text { years, end- } \\
\text { stage kidney } \\
\text { disease on } \\
\text { hemodialysis } \\
1 \text { year } \\
\text { +dexamethaso } \\
\text { ne } \\
\text { +remdesivir }\end{array}$ & $\begin{array}{l}\text { Pulmonary } \\
\text { mucormyco } \\
\text { sis }\end{array}$ & $\begin{array}{l}\text { Chest } \\
\text { radiograph, } \\
\text { CT chest, } \\
\text { matrix- } \\
\text { assisted laser } \\
\text { desorption/ion } \\
\text { ization } \\
\text { time of flight } \\
\text { (MALDI- } \\
\text { TOF), in vitro } \\
\text { antifungal } \\
\text { susceptibility } \\
\text { testing } \\
\text { (AFST) }\end{array}$ & $\begin{array}{l}\text { Rhizopus } \\
\text { microspo } \\
\text { rus }\end{array}$ & $\begin{array}{l}\text { Liposomal } \\
\text { amphoterici } \\
\text { n B }\end{array}$ & & Improved \\
\hline
\end{tabular}


Danish Javed et.al. Ayurveda and homeopathy in COVID associated mucormycosis.

\begin{tabular}{|c|c|c|c|c|c|c|c|c|c|c|}
\hline \multicolumn{11}{|c|}{ Table 1 Continued... } \\
\hline 7 & $\begin{array}{l}\text { Mehta } \\
\text { S, 2020 } \\
\text { (13) }\end{array}$ & 1 & $\begin{array}{lr}60- & \text { year-old } \\
\text { male } & \text { patient } \\
\text { bilateral } & \text { lid } \\
\text { edema } & \text { with } \\
\text { right } & \text { eye } \\
\text { prominence } & \end{array}$ & $\begin{array}{l}\text { DM } \\
\text { +oraloseltami } \\
\text { vir, } \\
\text { +iv } \\
\text { methylprednis } \\
\text { olone, } \\
\text { +dexamethaso } \\
\text { ne } \\
\text { +tocilizumab }\end{array}$ & $\begin{array}{l}\text { Rhino- } \\
\text { Orbital } \\
\text { Mucormyco } \\
\text { sis }\end{array}$ & $\begin{array}{l}\text { MRI brain, } \\
\text { orbits, and } \\
\text { paranasal } \\
\text { sinuses, nasal } \\
\text { biopsy }\end{array}$ & $\begin{array}{l}\text { Rhizopus } \\
\text { species }\end{array}$ & $\begin{array}{l}\text { Amphoteric } \\
\text { in B }\end{array}$ & $\begin{array}{l}\text { Due to poor } \\
\text { vitals } \\
\text { debridement } \\
\text { could not be } \\
\text { done }\end{array}$ & Death \\
\hline 8 & $\begin{array}{l}\text { Amirre } \\
\text { za V, } \\
2021 \\
(14)\end{array}$ & 2 & $\begin{array}{l}\text { 40-year old } \\
\text { woman with } \\
\text { bilateral visual } \\
\text { loss and } \\
\text { complete } \\
\text { ophthalmoplegia } \\
\text { of the right eye } \\
\text { and 54-year old } \\
\text { man with vision } \\
\text { loss, proptosis, } \\
\text { orbital } \\
\text { inflammation, } \\
\text { and complete } \\
\text { ophthalmoplegia } \\
\text { on the left side. }\end{array}$ & $\begin{array}{l}\text { Remdesivir } \\
\text { +dexamethaso } \\
\text { ne (both), } \\
\text { DM (male) }\end{array}$ & $\begin{array}{l}\text { Rhino- } \\
\text { orbital } \\
\text { mucormyco } \\
\text { sis }\end{array}$ & $\begin{array}{l}\text { CT scan, } \\
\text { MRI, } \\
\text { endoscopic } \\
\text { sinus } \\
\text { examination, } \\
\text { HPE }\end{array}$ & $\begin{array}{l}\text { Rhizopus } \\
\text { species }\end{array}$ & $\begin{array}{l}\text { Amphoteric } \\
\text { in } \mathrm{B} \text {, oral } \\
\text { posaconazol } \\
\mathrm{e}\end{array}$ & $\begin{array}{l}\text { Daily } \\
\text { endoscopic } \\
\text { sinus } \\
\text { debridement } \\
\text { and irrigation } \\
\text { with diluted } \\
\text { amphotericin } \\
\text { B. }\end{array}$ & $\begin{array}{l}\text { 1. Death } \\
\text { 2. Alive }\end{array}$ \\
\hline 9 & $\begin{array}{l}\text { Revann } \\
\text { avar } \\
\text { SM } \\
\text { etal, } \\
2021 \\
(15)\end{array}$ & 1 & $\begin{array}{l}\text { Middle-aged } \\
\text { woman, mild } \\
\text { COVID } 19 \\
\text { symptoms with } \\
\text { left-sided facial } \\
\text { pain, } \\
\text { ophthalmoplegia } \\
\text { of the left eye } \\
\text { and fever of } \\
\text { short duration. }\end{array}$ & Diabetes & $\begin{array}{l}\text { Orbital } \\
\text { apex } \\
\text { syndrome } \\
\text { with brain } \\
\text { infarction }\end{array}$ & $\begin{array}{l}\text { CT paranasal } \\
\text { sinus, MRI } \\
\text { brain, } \\
\text { HPE,Fungal } \\
\text { culture }\end{array}$ & $\begin{array}{l}\text { Rhizopus } \\
\text { species }\end{array}$ & $\begin{array}{l}\text { Amphoteric } \\
\text { in B }\end{array}$ & FESS & Improved \\
\hline 10 & $\begin{array}{l}\text { Chenna } \\
\text { mchett } \\
\text { y VK } \\
\text { etal, } \\
2021 \\
(16)\end{array}$ & 1 & $\begin{array}{l}\text { 72-year-old } \\
\text { male H/o severe } \\
\text { COVID } 19 \\
\text { pneumonia, c/o } \\
\text { streaky } \\
\text { hemoptysis }\end{array}$ & $\begin{array}{l}\text { hypertensive, } \\
\text { steroid- } \\
\text { induced } \\
\text { diabetic, } \\
\text { hypothyroid } \\
\text { +Ramdevpir } \\
\text { +methylpredn } \\
\text { isolone } \\
\text { +convalescent } \\
\text { plasma }\end{array}$ & $\begin{array}{l}\text { Pulmonary } \\
\text { mucormyco } \\
\text { sis }\end{array}$ & $\begin{array}{l}\text { PET CT, CT } \\
\text { guided } \\
\text { biopsy, HPE }\end{array}$ & $\begin{array}{l}\text { Rhizopus } \\
\text { species }\end{array}$ & $\begin{array}{l}\text { Liposomal } \\
\text { amphoterici } \\
\text { n B , } \\
\text { posaconazol } \\
\text { e }\end{array}$ & & Improved \\
\hline 11 & $\begin{array}{l}\text { Ehrenr } \\
\text { eich } \\
\text { AW, } \\
2020 \\
(17)\end{array}$ & 1 & $\begin{array}{l}33 \text {-year-old } \\
\text { female with } \\
\text { altered mental } \\
\text { status, } \\
\text { ophthalmoplegia } \\
\text { left } \quad \text { eye, } \\
\text { proptosis }\end{array}$ & $\begin{array}{l}\text { hypertension, } \\
\text { asthma }\end{array}$ & $\begin{array}{l}\text { Rhino- } \\
\text { orbital- } \\
\text { cerebral } \\
\text { mucormyco } \\
\text { sis }\end{array}$ & $\begin{array}{l}\text { CT head, } \\
\text { chest } \\
\text { radiograph }\end{array}$ & $\begin{array}{l}\text { Rhizopus } \\
\text { species }\end{array}$ & $\begin{array}{l}\text { Amphoteric } \\
\text { in B }\end{array}$ & $\begin{array}{l}\text { Refused by } \\
\text { family }\end{array}$ & Death \\
\hline 12 & $\begin{array}{l}\text { Pauli } \\
\text { MA. et } \\
\text { al, } \\
2021 \\
(18)\end{array}$ & 1 & $\begin{array}{l}50 \text {-year-old } \\
\text { female with } \\
\text { mild COVID } 19 \\
\text { symptoms and } \\
\text { painful lesion in } \\
\text { hard palate }\end{array}$ & $\begin{array}{l}\text { type } \\
\text { diabetes } \\
+ \text { Removable } \\
\text { partial denture }\end{array}$ & $\begin{array}{l}\text { Painful } \\
\text { palatal } \\
\text { lesion due } \\
\text { to } \\
\text { mucormyco } \\
\text { sis }\end{array}$ & $\begin{array}{l}\text { Incisional } \\
\text { biopsy, cone } \\
\text { beam } \\
\text { computed } \\
\text { tomography } \\
\text { scan. }\end{array}$ & $\begin{array}{l}\text { Rhizopus } \\
\text { species }\end{array}$ & $\begin{array}{l}\text { Amphoteric } \\
\text { in B }\end{array}$ & $\begin{array}{l}\text { Superficial } \\
\text { debridement }\end{array}$ & Improved \\
\hline 13 & $\begin{array}{l}\text { Sharma } \\
\text { S, et } \\
\text { al,2021 } \\
(19)\end{array}$ & 23 & $\begin{array}{l}15 \text { male, } 8 \\
\text { female old or } \\
\text { fresh COVID } 19 \\
\text { positive }\end{array}$ & $\begin{array}{l}\text { Diabetes } \\
\text { mellitus(21), } \\
\text { HTN(12), } \\
\text { Renal } \\
\text { Failure(1) }\end{array}$ & $\begin{array}{l}\text { Invasive } \\
\text { fungal } \\
\text { sinusitis } \\
\text { (ethmoids } \\
\text { (23), } \\
\text { Maxillary } \\
\text { (12), } \\
\text { sphenoid } \\
\text { (5), Frontal } \\
\text { (1)), Intra- } \\
\text { orbital } \\
\text { extension } \\
\text { (10), } \\
\text { intracranial } \\
\text { extension } \\
(2), \text { Palate } \\
\text { (9) }\end{array}$ & CT, MRI & $\begin{array}{l}\text { Not } \\
\text { mentione } \\
d\end{array}$ & $\begin{array}{l}\text { Amphoteric } \\
\text { in B }\end{array}$ & $\begin{array}{l}\text { Not } \\
\text { mentioned }\end{array}$ & $\begin{array}{l}\text { Not } \\
\text { mentioned }\end{array}$ \\
\hline
\end{tabular}




\begin{tabular}{|c|c|c|c|c|c|c|c|c|c|c|}
\hline \multicolumn{11}{|c|}{ Table 1 Continued... } \\
\hline 14 & $\begin{array}{l}\text { Sungur } \\
\text { teki H, } \\
\text { et al, } \\
2021(2 \\
0)\end{array}$ & 1 & $\begin{array}{l}\text { 56-year-old } \\
\text { female COVID } \\
19 \text { patient with } \\
\text { proptosis in the } \\
\text { right eye, } \\
\text { restricted eye } \\
\text { movements, } \\
\text { edema and color } \\
\text { change in the } \\
\text { nasal } \\
\text { area }\end{array}$ & $\begin{array}{l}\text { Diabetes } \\
+ \text { MPS }\end{array}$ & $\begin{array}{l}\text { Severe } \\
\text { Rhinocerebr } \\
\text { alMucormy } \\
\text { cosis }\end{array}$ & $\begin{array}{l}\text { Biopsy and } \\
\text { culture, } \\
\text { Paranasal } \\
\text { sinus CT, }\end{array}$ & $\begin{array}{l}\text { Rhizopus } \\
\text { species, } \\
\text { Acinetob } \\
\text { acterbau } \\
\text { mani }\end{array}$ & $\begin{array}{l}\text { Liposomal } \\
\text { amphoterici } \\
\mathrm{n} \quad \mathrm{B}, \\
\text { Colimicin }\end{array}$ & $\begin{array}{l}\text { Debridement, } \\
\text { Tracheostomy }\end{array}$ & Death \\
\hline
\end{tabular}

We found total 16 search results; out of which one (1) was a letter to the editor and one (1) was a review article. Remaining 14 articles were included in this study. Included twelve (12) articles were case studies, while two (2) were case series. Total forty six (46) patients of different types of mucormycosis were included in these search results. Cases of Sino-orbital, Rhino-sinusitis, Rhino-orbital, Rhinocerebral, Rhino-orbital-cerebral, Intensive fungal sinusitis, Orbital apex syndrome, Brain infection and painful palatal lesion were the presentation of mucormycosis. (Please see Table 1: Details of Case Reports/Series of mucormycosis) [7-20]

In these patients, gender-wise $65.2 \%$ (30) male and $34.8 \%$ female (16) are seen. Mean age was found 49.9 years age (38-78 years). In this group, diabetes was highly prevalent in $84.8 \%$ cases. One patient had type-1 diabetes, while one patient had developed steroid induced. Mucormycosis was seen to highly affect sinuses $(54.34 \%)$, then nasal part, eyeball, brain (34.8\% each), palate $(21.74 \%)$ and lungs $(6.5 \%)$.

The most common presenting complaints were eye swelling and pain, periorbital edoema, lid edoema, restricted eye movements, orbital inflammation, eye prominence, visual loss, complete ophthalmoplegia, proptosis, facial swelling, pain, numbness, nasal blockage, edoema and colour change in the nasal area, erythema headache, altered mental status, and painful palatal lesion.

Concerning investigations, in Rhinoorbital-cerebral mucormycosis cases, nasal endoscopy, nasal tissue biopsy, PNS CT, MRI brain orbits and paranasal sinuses, histopathological examination and fungal smear, PCR and DNA sequencing, matrixassisted laser desorption/ionization time of flight (MALDI-TOF), and in vitro antifungal susceptibility testing (AFST) were performed while in cases of pulmonary mucormycosis, CT Chest, Bronchoscopy, Endobronchial biopsy, and bronchoalveolar lavage (BAL), PET CT, CT guided biopsy, and HPE were performed. In the case of the palatal lesion, an incisional biopsy and cone beam computed tomography scan were performed. Rhizopusoryzae, R. microsporus, and other species were mainly recognized in microscopic smears in the form of fungal hyphae. Candida albicans, glabrata, and krusei along with Aspergillus flavus and niger were also encountered in one case of pulmonary mucormycosis.

Injection Fluconazole, Injection Amphotericin B, Injection Liposomal Amphotericin B, Voriconazole, Micafungin, Oral Posaconazole, Colimicinetc were used as primary antifungal agents in various cases. To prevent disease progression, functional endoscopic sinus surgery (FESS), surgical debridement, daily endoscopic sinus debridement and irrigation with diluted amphotericin B, orbital exenteration, endoscopic maxillectomy, ethmiodectomy, partial septectomy, bilateral maxillary antrostomy, total ethmoidectomy and sphenoidotomy, and more extensive surgeries were performed in these cases. Many patients refused surgical procedures, and in some cases, surgery was unable to be performed due to low vitals and technical difficulties. Mortality of available data was $39.13 \%$, whereas $60.87 \%$ cases improved with few deformities.

: An alternative and integrative strategy to mucormycosis 


\subsection{1 : Ayurveda perspective [21, 22]}

No such description

mucormycosis is found anywhere in Ayurveda text. However, diseases of head, neck, eyes and mouth are described deliberately in Ayurveda literature. Many symptoms of mucormycosis have close similarity with symptoms as described in Ayurveda. Covid associated opportunistic fungal infection is a result of a weak immune system. In this regard, we may treat the Covid disease on the line of Rajyakshama and Urdhva-jatrugata roga both simultaneously. Rajyakshama has similarity with the disease of the respiratory system which ultimately leads toward poor immune-compromised state (Oja Kshaya) of patients. So, in whole scenario, if we join the different pieces of jig-saw puzzle, the picture of Covid and its complications will be clear under the preview of Ayurveda by including Rajyakshama, Oja Kshaya, Siroroga, netraroga, nasarog and mukha rog.

In most of these conditions, which are found quite similar to Covid associated mucormycosis are showing preponderance of Vata and Pitta dosha exacerbation and Rakta and Mamsa dhatu are most affected dushya. Akash and vayu mahabhut are mainly affected in sinus and nasal disorders. Substantial Oja kshaya in post covid patients results in palpitation, anxiety, overthinking and pain all over the body. In the above condition, apara-oja from all over the body gets emaciated by three means i.e. ojavistransa, ojavyapada and ojakshaya. This course leads to symptoms like pain in joints, debility to do work, heaviness and stiffness in body, discolouration, laziness, giddiness, muscle wasting, weight loss, vertigo, delirium and all of sudden death. Here, mainly vata aggravation and immune compromised (vyadhi kshamatva or oja kshaya) conditions produce vicious events in patients (SU.SU 15/28-33 \& CH.Su 17/73).

In Krimijanya Shiroroga (pathological headache), Udarstha krimi (some microbes) are increased due to various reasons and causes reflex headache and blood mixed pus (Rakta and Puya) comes out from the nose. As per the Charaka, in case of krimijanya or pathological headache, Rakta and Mamsa dhatu get vitiated and cause Tridosha Prakopa which leads to the symptoms like Fever, Nasal discharge and Pain over sinuses and teeth (SU.U 25/10 \&CH.Chi 26/118). Here, headache is seen to be associated with krimi or microbes and the further progression of disease is always resulting in several complications related to eyes, sinus, ear and brain. In nasal disorders like "Apinasa ,Aampinasa Nasapuya, vyadhirakta and Dusthapinasa", results dryness, blockage, discharge and pain in nose, eye diseases, blurring vision, facial swelling and problem in functions of respiratory system blood tinged nasal discharge (SU.U 22/6, 22/17 \& 24/15-17 \&CH.Chi 26/114, 26/116). Symptoms like difficulty in closing of eyes, periorbital swelling, pain in eyeball, discharge, heaviness, blurring of vision redness and discoloration around eyes are seen in Vartmabandha,

Raktaabhishyanda, Klinnavartma, Sashofapaka, Amyladhyushita, Sirotpata, Sirapraharsha etc described by Sushruta and charaka (SU.U 3/17, 21 SU.U 6/9,28-30\&CH.Chi 26/129). Please see Table 2: Symptoms of mucormycosis compared with symptoms of Ayurvedic diseases

3.2.1.1 : Recommendations: Please see Table 3: Recommendation of ayurveda management

Table 2: Symptoms of mucormycosis compared with symptoms of Ayurvedic disease

\begin{tabular}{|c|c|c|c|}
\hline Mucormycosis symptoms & Symptoms in ayurveda & As per Sushruta samhita & As per Charaka samhita \\
\hline $\begin{array}{l}\text { Pain around eyes } \\
\text { Redness around eyes } \\
\text { Difficulty in closing of eyes }\end{array}$ & $\begin{array}{l}\text { Toda -needle pricking } \\
\text { pain } \\
\text { Lalima } \\
\text { present }\end{array}$ & $\begin{array}{l}\text { Vartambhandha-Su.U.3/17, } \\
\text { klinnvartama- Su. U. } 3 / 21, \\
\text { Sashofa paka, Su.U.6/21 } \\
\text { Raktaabhishyanda Su.U.6/9, } \\
\text { Amlyadushita Su.U.6/28 } \\
\text { Siroutpata \& Sirapraharsha Su.U.6/29\&30 } \\
\text { vartambhandha }\end{array}$ & $\begin{array}{l}\text { Vataj netraroga \& pittaj } \\
\text { netraroga } \\
\text { Ch.chi.26/129 }\end{array}$ \\
\hline
\end{tabular}


Danish Javed et.al. Ayurveda and homeopathy in COVID associated mucormycosis.

\begin{tabular}{|c|c|c|c|}
\hline \multicolumn{4}{|c|}{ Table 2 Continued... } \\
\hline $\begin{array}{llll}\begin{array}{l}\text { Blurring } \\
\text { diplopia }\end{array} & \text { of vision and } \\
\end{array}$ & & $\begin{array}{l}\text { Sirapraharsha, Su.U.6/30, } \\
\text { Dusthpratishayaupdrava, Su.u.24/17 }\end{array}$ & \\
\hline Periorbital swelling & Shofa/shotha & $\begin{array}{l}\text { Vartambhandha, klinnvartama, } \\
\text { Sashofa paka }\end{array}$ & $\begin{array}{l}\text { Krimajshirashoola } \\
\text { Ch.su.17/29 }\end{array}$ \\
\hline $\begin{array}{l}\text { Conjunctival infection and } \\
\text { chemosis }\end{array}$ & Kshta, vrana & $\begin{array}{l}\text { Vartambhandha, sashofapaka, } \\
\text { Arjuna -Su.U.4/7 }\end{array}$ & \\
\hline Headache & Ugraruja, Ghorruja & $\begin{array}{l}\text { Kshayajshiroroga, Su.u.25/9, } \\
\text { Krimijanyashiroroga } 25 / 10, \\
\text { Anantvata, } 25 / 13, \\
\text { Ardhavabheda, Su.u.25/15, } \\
\text { Nasapuyarakta, Su.u.22/10, }\end{array}$ & $\begin{array}{l}\text { Krimajshirashoola, } \\
\text { Ch.su.17/29, } \\
\text { Vatajshirashoola, } \\
\text { Ch.su.17/19-21, } \\
\text { Vatajpratishaya } \\
\text { sannipatajapratishaya } \\
\text { Ch.chi.26/105-106, } \\
\text { Sahasjanyarajyakshma, } \\
\text { Ch.ni.6/4 }\end{array}$ \\
\hline Bloody vomits/ haemoptysis & $\begin{array}{l}\text { Mukha se rakta } \\
\text { vaman/strava }\end{array}$ & $\begin{array}{l}\text { Yakshmashadrupa, Su.u.41/13, } \\
\text { Urshatajanyashosha, Su.u.41/29-31 }\end{array}$ & $\begin{array}{l}\text { Puyarakta, Ch.chi.26/116, } \\
\text { Sahasjanyarajyakshma, } \\
\text { Ch.ni.6/4, } \\
\text { Kshayajanyarajaykshma, } \\
\text { Ch.ni.6/8 } \\
\end{array}$ \\
\hline $\begin{array}{l}\text { Nasal discharge-blackish or } \\
\text { blood tinged } \\
\text { Nasal crusting }\end{array}$ & $\begin{array}{l}\text { Nasa se rakta and } \\
\text { puyastrava, } \\
\text { Shukhaourna abhasa }\end{array}$ & $\begin{array}{l}\text { Krimijanyashiroroga, } \\
\text { Nasapuyarakta, Su.u.22/10, } \\
\text { Nasashosha, Su.u.22/17, } \\
\text { Raktajanyapratishaya, Su.u.24/13, } \\
\text { Apanisha, Su.u.22/6, } \\
\text { Aampinasa, Su.u.24/15vimarsha, } \\
\text { Dusthpratishayaupdrava, Su.u.24/17 }\end{array}$ & $\begin{array}{l}\text { Apinasa, Ch.chi.26/114, } \\
\text { Puyarakta, Ch.chi.26/116, } \\
\text { Sahasjanyarajyakshma, } \\
\text { Ch.ni.6/4 }\end{array}$ \\
\hline $\begin{array}{l}\text { Blackish discolouration of } \\
\text { skin over nasolabial groove }\end{array}$ & & & Putinasya, Ch.chi.26/113 \\
\hline $\begin{array}{l}\text { Paraesthesia over half of face } \\
\text { Facial pain }\end{array}$ & $\begin{array}{l}\text { Ardhangghata } \\
\text { Ekangghata }\end{array}$ & $\begin{array}{l}\text { Anantvata, Su.u.25/14, } \\
\text { Ardhavabheda, Su.u.25/15 }\end{array}$ & \\
\hline $\begin{array}{l}\text { Pain over sinuses } \\
\text { Pain in teeth and gums }\end{array}$ & Dantashoola & Krimijanyashiroroga 25/10vimarsha, & \\
\hline Fever & Jwara & $\begin{array}{l}\text { Aampinasa, Su.u.24/15vimarsha, } \\
\text { Yakshmashadrupa, Su.u.41/13 }\end{array}$ & $\begin{array}{l}\text { Sahasjanyarajyakshma, } \\
\text { Ch.ni.6/4 }\end{array}$ \\
\hline $\begin{array}{l}\text { Loosening of teeth/ } \\
\text { discolouration of palate }\end{array}$ & Talushosha & Rajyakshamapurvaroopa, Su.u.41/11 & \\
\hline $\begin{array}{l}\text { Shortness of breath } \\
\text { Chest pain } \\
\text { Worsening of respiratory } \\
\text { symptoms }\end{array}$ & $\begin{array}{l}\text { Uroghata } \\
\text { Ushwasawrodha } \\
\text { Urshata }\end{array}$ & $\begin{array}{l}\text { Nasashosha, Su.u.22/17, } \\
\text { Raktajanyapratishaya, Su.u.24/13, } \\
\text { Dusthpratishayaupdrava, Su.u.24/17, } \\
\text { Rajyakshamapurvaroopa, Su.u.41/11, } \\
\text { Yakshmashadrupa,Su.u.41/13, } \\
\text { Urshatajanyashosha, Su.u.41/29-31, } \\
\text { Shokashoshi, Su.u.41/23 }\end{array}$ & $\begin{array}{l}\text { Sahasjanyarajyakshma,Ch.ni } \\
.6 / 4, \\
\text { Kshayajanyarajaykshma, } \\
\text { Ch.ni.6/8 }\end{array}$ \\
\hline $\begin{array}{l}\text { Altered mental status } \text { or } \\
\text { consciousness }\end{array}$ & $\begin{array}{l}\text { Brahm } \\
\text { Mada }\end{array}$ & $\begin{array}{l}\text { Ardhavabheda, Su.u.25/15, } \\
\text { Aampinasa, Su.u.24/15vimarsha, } \\
\text { Rajyakshamapurvaroopa, Su.u.41/11 }\end{array}$ & Putinasya, Ch.chi.26/113 \\
\hline
\end{tabular}

Table 3: Recommendation of ayurveda management

\begin{tabular}{|c|c|}
\hline General - (Su.u. 26/25-31; 41/35, 41; 24/18; 23/3,6; 10/5) & 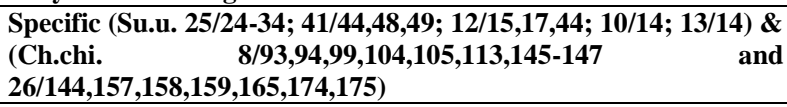 \\
\hline $\begin{array}{l}\text { Brimhanvidhi, Vata and Pitta nashakaushadhi, Madhurdravyas, } \\
\text { Kshaya and Kasanashakaushadi, Kriminashakaushadi }\end{array}$ & Trikatu, chavya and vidangachurna with ghrita and madhu, \\
\hline \multirow{3}{*}{$\begin{array}{l}\text { Ghritapana, Mandushna and MadhurDugdhapaan, goat and sheep } \\
\text { milk and ghee, Mamsarasa, ksheersarpi, ksheeranavikriti like } \\
\text { malai, ghevar,halua, lapsi etc. }\end{array}$} & $\begin{array}{lll}\text { AshwagandhadiChurna, } & \text { SitopladiChurna, } & \text { TalishadiChurna, } \\
\text { NagbalaChurna with milk } & & \\
\end{array}$ \\
\hline & MuktaPisthi, PravalPisthi \\
\hline & $\begin{array}{lll}\text { Vardhmanpippali, } & \text { ShilajeetVati, } & \text { Mahalaxmivilas } \\
\text { Saptaamritalauha }\end{array}$ \\
\hline \multirow[t]{5}{*}{ Shiralepa with vata and pitta nashakdravyas } & Vidangadichurna, vidangadikwatha \\
\hline & $\begin{array}{l}\text { Shadbindu tail, anu tail, balatail ,ksheersarpi, pradhmanchurna, } \\
\text { rasnadi tail for nasya(nasal instillation) }\end{array}$ \\
\hline & $\begin{array}{l}\text { Chandrodyavarti,Nagaarjunavarti,Yoganjana, jatipushpaanjana, } \\
\text { rasanjana, lekhayanjna, takshryaanjana,mustadyaanjanaforanjana }\end{array}$ \\
\hline & $\begin{array}{l}\text { TrifalaGhrita, Tilwakaghrita, Puranghrita, Dashmooladyaghrita, } \\
\text { Rasnaghrita, Balaghrita, Panchpanchmoolghrita, Vasa ghrita } \\
\text {,Shatavarighrita, Jivantiyadighrita, Mayurghrita, Mahamayurghrita, } \\
\text { KaumbhagritaforGhritapana }\end{array}$ \\
\hline & Trivrutghrit for mriduvirechana. \\
\hline
\end{tabular}

\subsection{2 : Homoeopathic Perspective}

Mucormycosis is no exception to the fact that homoeopathy is a medical system 
based on individualisation; each case must be presented in an individualistic manner.

Mucormycosis, or black fungus, can affect the sinuses, eyes, and brain and can occur concurrently with COVID-19 infection or in the early post-recovery phase. Even though mucor is present in the oral and respiratory linings of healthy people, it does not cause infection if the immune system is intact and healthy. In general, this infection occurs in patients with uncontrolled diabetes, undergoing cancer treatment, on long-term steroid therapy, following an organ transplant, and those who have suffered a severe injury such as a burn.

A large number of experimental studies have been carried out to assess the efficacy of homoeopathic drugs against fungal diseases in animals and humans [2327]. However, there have been many studies on fungal diseases in general, but as of our most recent search, we could not find any in-vitro/vivo or human trial studies on Mucormycosis.
Recently based on literature some anecdotal evidence released in the public interest by state authorities recommending mucormycosis treatment [28]. They proposed treatment for four types of mucormycosis: rhino-orbito-cerebral, pulmonary, cutaneous, and gastrointestinal.

For Rhino-orbito-cerebral Mucormycosis (ROCM), drug prescribed is ArsenicAlb, Kali Bich, Merc.Iod. Rubrum, Merc. Iod. Flavum, Merc. Sol, Cinnabaris, Thuja, Carbo Animalis; 200 potency, daily two times 6 pills each time for 5 days. ArsenicAlb, Phosphorous, Bryonia, Carbo Animalis, Ant.Tart; 200 potency, daily two times 6 pills each time for 5 days has been advised for Pulmonary mucormycosis (PM).

Likewise, in case of Cutaneous Mucormycosis (CM), it is Arsenic-Alb, Sulphur, Merc. sol, Anthracinum; 200 potency, daily two times 6 pills each time for 5 days while for Gastrointestinal Mucormycosis (GM), it is Arsenic-Alb, Phosphorous, Nitric Acid; 200 potency, daily two times 6 pills each time for 5 days.

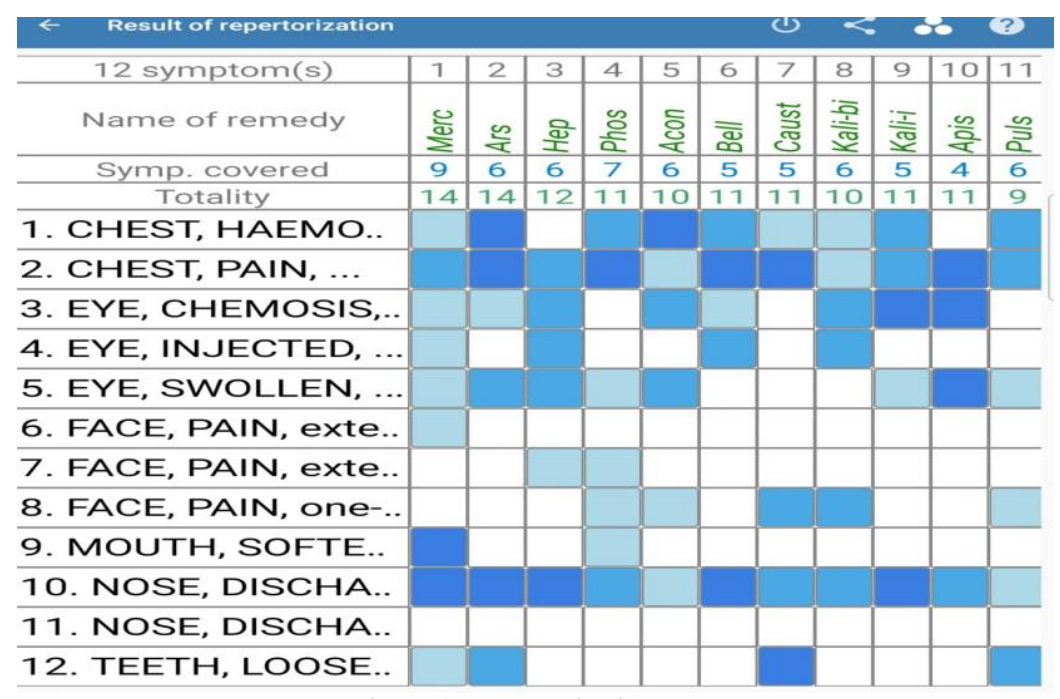

Figure 1: Repertorisation sheet

Another advisory for homoeopathic practitioners, though not based on any specific trial, has been released for the management of mucormycosis by the Drug Policy Section of the Ministry of AYUSH, Government of India, in consultation with senior physicians and researchers [29].
Based on symptoms guided by ICMR, repertorisation was carried out with the help of Homoeopathic Repertorium [30] and details of rubrics considered for repertorisation and results are being given. (Please see-Figure 1: Repertorisation sheet) and (See-Table 4: Details of Repertorisation sheet with medicines) 
Table 4: Details of Repertorisation sheet with medicines

\begin{tabular}{|c|c|c|c|}
\hline $\begin{array}{l}\text { S. } \\
\text { No. }\end{array}$ & Rubrics & \multicolumn{2}{|l|}{$\begin{array}{l}\text { Medicines (Short name \| full name) with } \\
\text { S-Symptoms covered, } \\
\text { R-Total marks }\end{array}$} \\
\hline 1. & CHEST, HAEMORRHAGE, $\ldots$ & \multirow{12}{*}{ 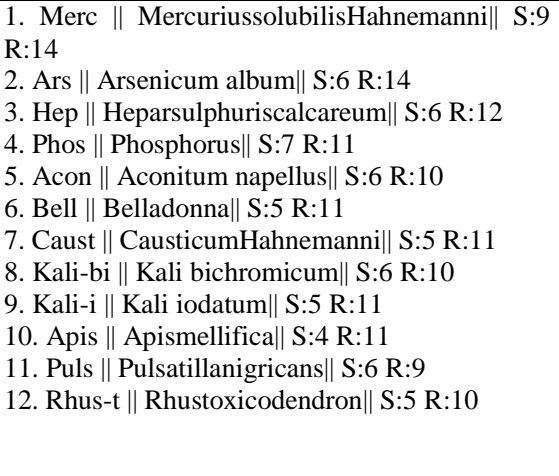 } & \multirow{12}{*}{$\begin{array}{l}\text { 13. Bry } \| \text { Bryonia alba\| S:5 R:9 } \\
\text { 14. Ferr } \| \text { Ferrummetallicum\| S:5 R:9 } \\
\text { 15. Ferr-ar } \| \text { Ferrumarsenicosum\| S:5 } \\
\text { R:9 } \\
\text { 16. Lach } \| \text { Lachesismuta\| S:5 R:9 } \\
\text { 17. Nux-v } \| \text { Nux vomical| S:5 R:9 } \\
\text { 18. Kreos } \| \text { Kreosotum\| S:5 R:8 } \\
\text { 19. Mez } \| \text { Mezereum\| S:5 R:8 } \\
\text { 20. Nit-ac } \| \text { Nitricumacidum\| S:4 R:9 } \\
\text { 21. Am-c } \| \text { Ammonium carbonicum } \| \text { S:4 } \\
\text { R:8 } \\
\text { 22. Con } \| \text { Conium maculatum\| S:5 R:7 } \\
\text { 23. Ip } \| \text { Ipecacuanha\| S:4 R:8 } \\
\text { 24. Kali-c } \| \text { Kali carbonicum\| S:4 R:8 } \\
\text { 25. Sep } \| \text { Sepia succus } \| \text { S:4 R:8 }\end{array}$} \\
\hline 2. & CHEST, PAIN, . & & \\
\hline 3. & EYE, CHEMOSIS, ... & & \\
\hline 4. & EYE, INJECTED, ... & & \\
\hline 5. & EYE, SWOLLEN, & & \\
\hline 6. & FACE, PAIN, extending to, teeth & & \\
\hline 7. & $\begin{array}{l}\text { FACE, PAIN, extending to, } \\
\text { temples }\end{array}$ & & \\
\hline 8. & FACE, PAIN, one-sided & & \\
\hline 9. & MOUTH, SOFTENING, GUMS & & \\
\hline 10. & NOSE, DISCHARGE, bloody, ... & & \\
\hline 11. & NOSE, DISCHARGE, brownish & & \\
\hline 12. & TEETH, LOOSENESS, painful & & \\
\hline
\end{tabular}

Table 5:Indications of Homoeopathic medicines suggested for Mucormycosis $(31,32,33,34,35)$

\begin{tabular}{|c|c|c|c|}
\hline $\begin{array}{l}\text { S. } \\
\text { No. }\end{array}$ & $\begin{array}{l}\text { Types of } \\
\text { Mucormycosis }\end{array}$ & Medicines & Indications \\
\hline \multirow[t]{8}{*}{1.} & \multirow[t]{8}{*}{$\begin{array}{l}\text { Rhino-orbito- } \\
\text { cerebral }\end{array}$} & Arsenic Alb & $\begin{array}{l}\text { Hemicrania relieves by cold, Periodical burning pains, with restlessness; with cold skin. } \\
\text { Oedematous eyes, inflamed, with extreme painfulness; burning, hot, and excoriating } \\
\text { lachrymation. Thin watery excoriating discharge from nose, blocked feeling with burning and } \\
\text { bleeding. }\end{array}$ \\
\hline & & Kali Bich & $\begin{array}{l}\text { Septum ulcerated; round ulcer. Fetid smell. Discharge thick, ropy, and greenish-yellow. Tough, } \\
\text { elastic plugs from nose; leave a raw surface. Inflammation extends to frontal sinuses, with } \\
\text { distress and fullness at root of nose. Coryza, with obstruction of nose. Violent sneezing. Loss of } \\
\text { smell. Unilateral headache in small spots, and from suppressed catarrh. Frontal pain; usually } \\
\text { over one eye. }\end{array}$ \\
\hline & & $\begin{array}{l}\text { Merc.Iod. } \\
\text { Rubrum }\end{array}$ & $\begin{array}{l}\text { Coryza and dull hearing; right side of nose hot. Hawks mucus from posterior nares. Turbinated } \\
\text { bones swollen. Boggy mucus membrane of nose and throat; closure of Eustachian tube, opening } \\
\text { with a pop. }\end{array}$ \\
\hline & & $\begin{array}{l}\text { Merc. Iod. } \\
\text { Flavum }\end{array}$ & $\begin{array}{l}\text { Small ulcers on posterior pharynx. Easily detached patches on inflamed pharynx and fauces; } \\
\text { worse on right tonsil; much tenacious mucus. Constant inclination to swallow. }\end{array}$ \\
\hline & & Merc. Sol & $\begin{array}{l}\text { One-sided, tearing pains. Catarrhal headaches; much heat in head.Lids red, thick, swollen. } \\
\text { Profuse, burning, acrid discharge. }\end{array}$ \\
\hline & & Cinnabaris & $\begin{array}{l}\text { Severe shooting pain in bones of orbit, especially running from inner to outer canthus in the } \\
\text { bone. Redness of whole eye. Lids granulated; canthi and lids red.For ciliary neuralgia and } \\
\text { ulceration upon a syphilitic base. }\end{array}$ \\
\hline & & Thuja & $\begin{array}{l}\text { Catarrh; thick, green mucus; blood and pus. On blowing nose, pain in teeth. Ulceration within } \\
\text { the nostrils. Dryness of nasal cavities. Left-sided headache. }\end{array}$ \\
\hline & & $\begin{array}{l}\text { CarboAnim } \\
\text { alis }\end{array}$ & $\begin{array}{l}\text { Headache, as if head had been blown to pieces. Rush of blood with confusion. Sensation as if } \\
\text { something lay above eyes so that she could not look up. }\end{array}$ \\
\hline \multirow[t]{5}{*}{2.} & \multirow[t]{5}{*}{ Pulmonary } & Arsenic Alb & $\begin{array}{l}\text { Burning in chest. Suffocative catarrh. Cough worse after midnight; worse lying on back. } \\
\text { Expectoration scanty, frothy. Darting pain through upper third of right lung. Wheezing } \\
\text { respiration. Haemoptysis with pain between shoulders; burning heat all over. }\end{array}$ \\
\hline & & $\begin{array}{l}\text { Phosphoro } \\
\text { us }\end{array}$ & $\begin{array}{l}\text { Cough worse, cold air. Sweetish taste while coughing. Hard, dry, tight, racking cough. } \\
\text { Congestion of lungs. Burning pains, heat and oppression of chest. Tightness across chest; great } \\
\text { weight on chest. Sharp stitches in chest; respiration quickened, oppressed. Pneumonia, with } \\
\text { oppression; worse, lying on left side. Sputa rusty, blood-colored, or purulent. }\end{array}$ \\
\hline & & Bryonia & $\begin{array}{l}\text { Dry, hacking cough from irritation in upper trachea. Cough, dry, at night; must sit up; worse } \\
\text { after eating or drinking, with vomiting, with stitches in chest, and expectoration of rust-colored } \\
\text { sputa. Frequent desire to take a long breath; must expand lungs. Difficult, quick respiration; } \\
\text { worse every movement; caused by stitches in chest. Croupous and pleuro-pneumonia. }\end{array}$ \\
\hline & & $\begin{array}{l}\text { CarboAnim } \\
\text { alis }\end{array}$ & $\begin{array}{l}\text { Pleurisy, typhoid character, and remaining stitch. Ulceration of lung, with feeling of coldness of } \\
\text { chest. Cough, with discharge of greenish pus. }\end{array}$ \\
\hline & & Ant.Tart & $\begin{array}{l}\text { Great rattling of mucus, but very little is expectorated. Rapid, short, difficult breathing; seems as } \\
\text { if he would suffocate; must sit up. Oedema and impending paralysis of lungs. }\end{array}$ \\
\hline \multirow[t]{3}{*}{3.} & \multirow[t]{3}{*}{ Gastrointestinal } & Arsenic Alb & $\begin{array}{l}\text { Gastralgia; Burning pain in stomach,Great thirst; drinks much, but little at a time. Vomiting of } \\
\text { blood, bile, green mucus, or brown-black mixed with blood. }\end{array}$ \\
\hline & & $\begin{array}{l}\text { Phosphoro } \\
\text { us }\end{array}$ & $\begin{array}{l}\text { Pain in stomach; relieved by cold food, ices. Stomach painful to touch. Inflammation of } \\
\text { stomach, with burning extending to throat and bowels.Vomiting; water is thrown up as soon as it } \\
\text { gets warm in the stomach and Haematemesis. }\end{array}$ \\
\hline & & Nitric Acid & $\begin{array}{l}\text { Pain in cardiac orifice.Haemorrhages from bowels, profuse, bright. Diarrhœa, slimy and } \\
\text { offensive. After stools, irritable and exhausted. }\end{array}$ \\
\hline \multirow[t]{4}{*}{4.} & \multirow[t]{4}{*}{ Cutaneous } & Arsenic Alb & Ulcers with offensive discharge. Gangrenous inflammations. \\
\hline & & Sulphur & Itching, burning; worse scratching, washing, warmth and in evening. \\
\hline & & Merc. sol & $\begin{array}{l}\text { Glands swell every time patient takes cold. Buboes.Ulcers, irregular in shape, edges undefined. } \\
\text { Excessive odorous viscid perspiration; worse, night. }\end{array}$ \\
\hline & & $\begin{array}{l}\text { Anthracinu } \\
m\end{array}$ & $\begin{array}{l}\text { Ulceration, sloughing and intolerable burning.Haemorrhages, black, thick, tar-like, rapidly } \\
\text { decomposing. }\end{array}$ \\
\hline
\end{tabular}


Further, indications of these possible groups of medicines are given in consultation with different Materia Medica Books as directed from the abovementioned advisories and from repertorisation results. (Please see-Table 5:Indications of Homoeopathic medicines suggested for Mucormycosis ${ }^{31,32,33,34,35)}$ ]

\section{DISCUSSION}

Although no such robust study was found in our search, a study of the authentic books of Ayurveda and Homeopathy has revealed some possible medicine that can be used. However, the approach while treating such cases may be multiple. As reported, mucormycosis cases are having poor immunity and hyperglycemia, an integrated plan of treatment may be beneficial. A team from different departments of modern medicine and AYUSH is vastly required to treat these cases. Simultaneously, role of AYUSH in these cases must be well defined.

Complementary and alternative medicine (CAM) may delivers additive effect to standard treatment in the form of immunomodulation (rasayana), hyperglycemic control through diet and therapy, purification treatment (mild purgation, nasal cleaning, oral and throat cleaning), fumigation therapy, oil pooling treatment etc. Prevention, communication, motivational guidance, personal and environmental hygiene, yoga and meditation, supportive treatment to those who are not fit for surgery or refusing are the significant domains to be covered by AYUSH experts. A proper protocol may be framed to provide AYUSH care. Experts of different field can bring significant change if they work in coordination. Fumigation (dhupan) with dry herbal medicines; oral hygiene by neem, clove, turmeric, basil, maulshri etc; kawal and gandush for oral and pharyngeal cleaning; nasal cleaning by anu tail, shigru oil, vidanga oil etc.; eye care by Maha triphala ghrit, anjana etc. may be facilitated to all the post COVID-19 patients as a prophylactic measure. Use of fresh mask especially impregnated with herbal decoctions and essential oil may work as anti-microbial as well as mood elevators. Regular practice of slow deep breathing pranayama, bhramari, Shitali etc. will give add-on benefit on respiratory parameters.

Homoeopathy treatment is mainly based on symptoms; in this unwanted disease also the help of this AYUSH system can be taken. However, in our search, no such test was found directly related to this disease in which this medicine is infallible in its treatment, yet as it is known that if the homoeopathy medicines are discovered by keeping the symptoms at the centre, then it is possible to get favourable results. Symptoms of ROCM; Facial pain, headache, lethargy, visual loss, proptosis, and/or palatal ulcer, nasal discharge (blackish/bloody), blurred or double vision with pain may be combated chiefly through Arsenic album, Kali bichromicum and Merc solubilis. Difficult breathing, cough, chest pain, pleural effusion, haemoptysis, worsening of respiratory symptoms (PM) managed by Arsenic album, Phosphorus, Bryonia, Acalypha indica etc. Similarly, GM and CM can be combated with Arsenic album, Lachesis, Merc Corrosivus Phosphorus etc and Anthracinum, Arsenic album, Lachesis, Sulphur, Secale cornutum etc respectively [28-29, 31-35]. Homeopathic therapy is significantly less expensive than conventional pharmacological therapy [36]. Patient satisfaction is also higher in the homoeopathic group than in the conventional treatment group [37]. Homeopathic remedies are generally thought to be safe [38]. However, it is not without risk, and a few mild adverse events were reported in the studies reviewed [39].

\section{CONCLUSION}

In the absence of rigorous trials, we found no conclusive comment on the efficacy of CAM in mucormycosis, but anecdotal evidence offers possibilities of CAM. To demonstrate its role, we 
recommend that rigorous exploratory pilot randomised control trials be conducted in the future, either alone or as an add-on.

\section{Acknowledgement: None}

\section{Conflict of Interest: None}

\section{Source of Funding: None}

\section{REFERENCES}

1. Dhama K, Khan S, Tiwari R, Sircar S, Bhat S, Malik YS, Singh KP, Chaicumpa W, Bonilla-Aldana DK, Rodriguez-Morales AJ. Coronavirus Disease 2019-COVID-19. Clin Microbiol Rev. 2020 Jun 24;33(4):e0002820. doi: 10.1128/CMR.00028-20. PMID: 32580969 ; PMCID: PMC7405836.

2. Jeong W, Keighley C, Wolfe R, Lee WL, Slavin MA, Kong DCM, Chen SC. The epidemiology and clinical manifestations of mucormycosis: a systematic review and meta-analysis of case reports. Clin Microbiol Infect. 2019 Jan;25(1):26-34. doi: 10.1016/j.cmi.2018.07.011. Epub 2018 Jul 21. PMID: 30036666.

3. Skiada A, Pavleas I, Drogari-Apiranthitou M. Epidemiology and Diagnosis of Mucormycosis: An Update. J Fungi (Basel). 2020 Nov 2;6(4):265. doi: 10.3390/jof6040265. PMID: 33147877; PMCID: PMC7711598.

4. Prakash H, Chakrabarti A. Global epidemiology of mucormycosis. $\mathbf{J}$ Fungi2019;5:26.

5. Ayurvedic medicines for Black Fungus cases - The Hindu [Internet]. [cited 2021 May 21]. Available from: https://www.thehindu.com/news/cities/Hyde rabad/ayurvedic-medicines-for-blackfungus-cases/article34600886.ece

6. Telangana declares black fungus a notified disease | Cities News,The Indian Express [Internet]. [cited 2021 May 21]. Available from:

https://indianexpress.com/article/cities/hyde rabad/telangana-declares-black-fungus-anotified-disease-7322659/

7. Maini A, Tomar G, Khanna D, Kini Y, Mehta H, Bhagyasree V. Sino-orbital mucormycosis in a COVID-19 patient: A case report. Int J Surg Case Rep. 2021 May;82:105957. doi: 10.1016/j.ijscr.2021.105957. Epub 2021
May 4. PMID: 33964720; PMCID: PMC8093005.

8. Tabarsi P, Khalili N. COVID 19 associated rhinosinusitismucormycosis due to Rhizopusoryzae : A rare but potentially fatal infection occurring after treatment with corticosteroids. Research squire:1-11. DOI: https://doi.org/10.21203/rs.3.rs-398594/v1

9. Khan, N., Gutierrez, C., Villafuerte Martinez, D., \& Proud, K. (2021). A case report of COVID-19 associated pulmonary mucormycosis. Archive of Clinical Cases, 7(3), Arch Clin Cases 2020; 7(3):46-51. doi:http://dx.doi.org/10.22551/2020.28.0703 .10172

10. Mishra, N., Mutya, V., Thomas, A., Rai, G., Reddy, B., Mohanan, A., Ray, S., Thiruvengadem, A., Siddini, V., \& Hegde, R. (2021). A case series of invasive mucormycosis in patients with COVID-19 infection. International Journal of Otorhinolaryngology And Head And Neck Surgery, 7(5), 867-870. doi: http://dx.doi.org/10.18203/issn.24545929.ijohns20211583

11. Alekseyev K, Didenko L, Chaudhry B. Rhinocerebral Mucormycosis and COVID19 Pneumonia. J Med Cases. 2021 Mar;12(3):85-89. doi: 10.14740/jmc3637. Epub 2021 Jan 19. PMID: 33984095; PMCID: PMC8040444.

12. Garg D, Muthu V, Sehgal IS, Ramachandran R, Kaur H, Bhalla A, Puri GD, Chakrabarti A, Agarwal R. Coronavirus Disease (Covid-19) Associated Mucormycosis (CAM): Case Report and Systematic Review of Literature. Mycopathologia. 2021 May;186(2):289298. doi: 10.1007/s11046-021-00528-2. Epub 2021 Feb 5. PMID: 33544266; PMCID: PMC7862973.

13. Mehta S, Pandey A. Rhino-Orbital Mucormycosis Associated With COVID 19. Cureus. 2020;12(9):10-4. doi:10.7759/cureus. 10726

14. Veisi A, Bagheri A, Eshaghi M, Rikhtehgar MH, RezaeiKanavi M, Farjad R. Rhinoorbital mucormycosis during steroid therapy in COVID 19 patients: A case report. Eur J Ophthalmol. 2021; Article first published online: April 10, 2021

15. Revannavar SM, Supriya P, Samaga L, Vineeth K. COVID 19 triggering mucormycosis in a susceptible patient: A 
new phenomenon in the developing world? BMJ Case Rep. 2021;14(4).

16. Chennamchetty VK, Adimulapu S, Kola BP, Padua MD, Ambika C, Verma MK, Rao MVR. Post-COVID pulmonary mucormycosis- A case report. IP Indian $\mathbf{J}$ Immunol Respir Med. 2021; 6(1):62-66.

17. Werthman-Ehrenreich A. Mucormycosis with orbital compartment syndrome in a patient with COVID 19. Am J Emerg Med [Internet]. 2021;42(xxxx):264.e5-264.e8. Available from: https://doi.org/10.1016/j.ajem.2020.09.032

18. Pauli MA, Pereira L de M, Monteiro ML, de Camargo AR, Rabelo GD. Painful palatal lesion in a patient with COVID 19. Oral Surg Oral Med Oral Pathol Oral Radiol [Internet]. 2021;00(00). Available from: https://doi.org/10.1016/j.oooo.2021.03.010

19. Sharma S, Grover M, Bhargava S, Samdani S, Kataria T. Post coronavirus disease mucormycosis: A deadly addition to the pandemic spectrum. J Laryngol Otol. 2021;(May).

20. Sungurtekin H, Sargin F, Akbulut M, Karaduman S. Severe Rhinocerebral Mucormycosis Case Developed after COVID 19. J BacteriolParasitol $1 \quad \mathrm{~J}$ Bacteriol Parasitol [Internet]. 2021; 12(1):1000386. Available from: https://www.longdom.org/openaccess/severe-rhinocerebral-mucormycosiscase-developed-after-COVID 191961170.html\#ai

21. Sushruta Acharya, Sushruta Samhita edited with Ayurved tattva sandipika, Uttartantra, editor Kaviraja Ambika Dutta Shastri, Chaukhamba Sanskrit Sansthan, Varansi, Reprint 2017.

22. Charak Acharya, Charak Samhita edited with Vidyotni commentary, SutraSthana, Nidana Sthana \& Chikitsa Sthana, , editor Pt.Kasinatha Shastri \& Dr .Gorakha Natha Chaturvedi, Chaukambha Bharati Academy, Varansi, Reprint 2018.

23. Nagai MY, Dalboni LC, Cardoso TN, Correia MS, Pinto SAG, Pinto AAG, Coelho CP, Alvarez-Saraiva A, Peres GB, Lallo MA, Bonamin LV. Effects of Homeopathic Phosphorus on Encephalitozooncuniculi-Infected Macrophages In-Vitro. Homeopathy. 2019 Aug;108(3):188-200. doi: 10.1055/s-00391678700. Epub 2019 Apr 18. PMID: 30999383.
24. Prajapati S, Sharma M, Kumar A, Gupta P, Dwivedi B, Arya BS, Arya R, Nayak D. Antimicrobial activity of different homoeopathic drugs and their potencies against'Aspergillusniger'In vitro. Indian Journal of Research in Homoeopathy. 2019 Jul 1;13(3):150.

25. Gupta G, Srivastava A K, Gupta N, Gupta G, Mishra S. Anti-candidal activity of homoeopathic drugs: An in-vitro evaluation. Indian J Res Homoeopathy 2015;9:79-85

26. Gupta G, Garg KL, Chandra B. Effects of homoeopathic drugs against fungi isolated from human patients. Asian Homoeopath J 1995;5:15-8.

27. Gupta G, Srivastava AK, Chandra B, Gupta N. Mycoses: An update over clinical cure with homoeopathic drugs. Asian Homoeopath J 1997b; 7:67-96

28. Special correspondent. Ayush comes out with Homoeopathic treatment protocol for Black Fungus. THE HINDU.21/05/2021.Available at https://www.thehindu.com/news/cities/Hyde rabad/ayush-comes-out-with-homoeopathictreatment-protocol-for-black-

fungus/article34617981.ece.accessed on 27/05/2021

29. Drug Policy Section, Ministry of AYUSH, Government of India. Information for Homoeopathy Practitioners for Symptomatic Management of Suspected and Diagnosed cases of Mucormycosis Available at https://www.ayush.gov.in/docs/Information $\% 20$ for\%20homoeopathic\%20management $\% 20$ of\%20Mucormycosis.pdf accessed on $29 / 05 / 2021$

30. Homoeopathic Repertorium. Android version 3.9.5.7 developed by Soft Solutions available on play store.

31. Allen H.C. ; Keynotes and Characteristics with Comparison of some of the Leading remedies of the Materia Medica with Bowel Nosodes; Low price Edition; New Delhi; B. Jain Publishers (P) Ltd.; 2002: 47-295

32. Lippe A.; Keynote \& Redline Symptoms of Materia Medica ; Reprint edition; New Delhi; B. Jain Publishers(P)Ltd.; 2004: 35163

33. Hahnemann S.; Materia Medica Pura; Vol1; 16th impression; Translated by R.E. Dudgeon; New Delhi ; B. Jain Publishers(P)Ltd.;2010: 113-608 
34. Hahnemann S.; Materia Medica Pura; Vol2; 16th impression; Translated by R. E. Dudgeon; New Delhi; B. Jain Publishers(P)Ltd.; 2010: 144-649

35. Boericke W.; Pocket Manual of Homoeopathic Materia Medica\& Repertory; Low price edition; New Delhi; B. Jain Publishers (P) Ltd.; 2002: 79-645

36. Trichard M, Chaufferin G, Nicoloyannis N. Pharmacoeconomic comparison between homeopathic and antibiotic treatment strategies in recurrent acute rhinopharyngitis in children. Homeopathy 2005;94:3-9.

37. Malapane E, Solomon EM, Pellow J. Efficacy of a homeopathic complex on acute viral tonsillitis. J Altern Complement Med. 2014;20(11):868-873. doi:10.1089/acm.2014.0189
38. Dantas F, Rampes H. Do homeopathic medicines provoke adverse effects? a systematic review. $\mathrm{Br}$ Homeopath J. 2000;89(suppl 1):S35-S38.

39. Riley D, Fischer M, Singh B, Haidvogl M, Heger M. Homeopathy and conventional medicine: An outcomes study comparing effectiveness in a primary care setting. $\mathbf{J}$ Altern Complement Med 2001;7:149-59.

How to cite this article: Javed D, Dixit AK, Vats $\mathrm{H}$ et.al. Review of published case reports of COVID-19 associated mucormycosis with search of therapeutic potential in ayurveda and homeopathy. Int J Health Sci Res. 2021; 11(8): 114-126. DOI: https://doi.org/10.52403/ijhsr. 20210817 